\title{
The laryngeal mask airway in fibreoptic bronchoscopy
}

\author{
Alan R Smyth, Andrew R Bowhay, Louisa J Heaf, Rosalind L Smyth
}

\begin{abstract}
A large series of patients is reported in whom the laryngeal mask airway was used to perform fibreoptic bronchoscopy. It allows direct visualisation of the airways during spontaneous respiration. It has enabled bronchoalveolar lavage and bronchography to be performed in children as young as 6 months and transbronchial biopsies in children as young as 4 years. (Arch Dis Child 1996;75:344-345)
\end{abstract}

Keywords: fibreoptic bronchoscopy, laryngeal mask airway.

Fibreoptic bronchoscopy has been used successfully in children since the early $1980 \mathrm{~s}$. It allows the assessment of a number of respiratory problems including upper airways obstruction, lobar collapse, persistent wheeze, and opportunistic pneumonia. ${ }^{1}$ As well as direct visualisation of the airways, several procedures are possible through the fibreoptic bronchoscope. These include bronchoalveolar lavage, endobronchial biopsy, transbronchial biopsy, and bronchograms. ${ }^{2}$

In line with practice in many other units in the United Kingdom, we use general anaesthetic, rather than sedation, for all bronchoscopies. This enables better monitoring and airway control. It has been advocated that transbronchial biopsies in children should only be performed under general anaesthetic. ${ }^{2}$ If an endotracheal tube is used, it may not be possible to pass an appropriately sized bronchoscope through it. The smallest bronchoscope which has a biopsy channel is $5 \mathrm{~mm}$ in diameter and this must be passed through a endotracheal tube of at least $7 \mathrm{~mm}$ internal diameter. This would effectively mean that transbronchial biopsies could only be performed in children over 8 years of age. ${ }^{2}$ The smallest bronchoscope with a suction channel is $3.5 \mathrm{~mm}$ in diameter and requires an endotracheal tube of at least $5.5 \mathrm{~mm}$ in diameter. The lower age limit for bronchoalveolar lavage would then be 5 years.

The use of the laryngeal mask airway (LMA) in anaesthesia was first described by Brain in $1983 .^{3}$ The device consists of a tube, resembling a standard endotracheal tube, connected to an elliptical mask, which is inflated in the hypopharynx, making a seal around the larynx. The LMA currently manufactured (Colgate Medical, Windsor, Berkshire) is available with tubes of internal diameters of $7 \mathrm{~mm}, 8.4 \mathrm{~mm}$, and $10 \mathrm{~mm}$. The size 2 LMA (internal diameter $7 \mathrm{~mm}$ ) is suitable for use in babies

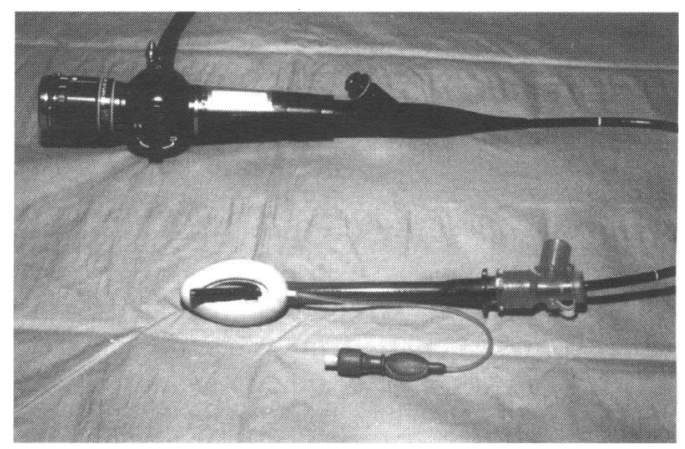

Figure 1 The $5 \mathrm{~mm}$ bronchoscope passing through a size 2 laryngeal mask airway.

and children up to $20 \mathrm{~kg}$ and will allow the passage of a $5 \mathrm{~mm}$ bronchoscope (fig 1). This allows transbronchial biopsy to be performed in much younger children.

We describe our experience of the LMA in a large series of children undergoing fibreoptic bronchoscopy and the associated diagnostic procedures performed.

\section{Methods}

The paediatric fibreoptic bronchoscopy service at Alder Hey was established in 1992. The number of procedures performed for each indication is summarised in table 1 . All procedures were performed under general anaesthesia, using either endotracheal intubation or an LMA. The two sizes of bronchoscope used had external diameters $5 \mathrm{~mm}$ (Olympus BF P20D, Keymed, Southend-on-Sea.) and $3.5 \mathrm{~mm}$ (Olympus BF 3C20). Anaesthetic and bronchoscopic techniques were as previously described. ${ }^{1}{ }^{4}$ No significant complications of the procedure were recorded during this period.

\section{Results}

We performed 151 fibreoptic bronchoscopies on 129 patients (median age 6 years, range 5 months to 17 years) of whom 54 (36\%) were girls. The LMA was used in $79(52 \%)$ bronchoscopies and its use has increased over time. When the service was established in 1992, only one in 18 bronchoscopies were performed using the mask. By 1995 this figure was 30/44. We performed 65 bronchoscopies on children under 5 years of whom $46(71 \%)$ had bronchoalveolar lavage $(42 / 46$ using the LMA).

Transbronchial biopsies were performed in nine patients, four of these using the LMA (median age 5 years, range 4.5 to 7.5 years) and five using endotracheal intubation (median age 15.2 years, range 7.5 to 17 years). 
Table 1 Indications for fibreoptic bronchoscopy in 151 patients

\begin{tabular}{ll}
\hline Indication & Number (\%) of patients \\
\hline Persistent collapsed lobe & $59(39)$ \\
Opportunistic pneumonia & $33(22)$ \\
Foreign body & $11(7)$ \\
Interstitial lung disease & $7(5)$ \\
Tracheomalacia/bronchomalacia & $7(5)$ \\
Follow up of bronchial carcinoid & $5(3)$ \\
Haemoptysis & $2(1)$ \\
Other & $27(18)$
\end{tabular}

^ Fibreoptic bronchoscopy performed in conjunction with rigid bronchoscopy.

(This included one child aged 7.5 years whose weight was on 97 th centile for age.) In addition $6 / 8$ bronchograms and 59/111 bronchoalveolar lavage procedures were performed using the LMA. In total 69/134 of all procedures were performed using the laryngeal mask.

\section{Discussion}

We have shown in this large series that the LMA provides a practical and safe anaesthetic technique for fibreoptic bronchoscopy in children. A recent report ${ }^{4}$ described the use of the LMA in six children undergoing fibreoptic bronchoscopy and called for further studies to define the role of this technique. The report did not describe the use of any procedures during these bronchoscopies.
Our report shows that the LMA offers advantages over endotracheal intubation where bronchoscopy with bronchoalveolar lavage or a bronchogram is needed in children under 5 years and where transbronchial biopsies are needed in children under 8 years. This may be particularly important for heart-lung transplant units where transbronchial biopsies are frequently performed. It also enables visualisation of the glottis, subglottis, and upper trachea and can be used in children who are breathing spontaneously to assess airway dynamics in the diagnosis of tracheomalacia or bronchomalacia.

The only contraindication to the use of the LMA for fibreoptic bronchoscopy is severe respiratory distress where endotracheal intubation and intermittent positive pressure ventilation offer a safer alternative.

1 Wood RE, Postema D. Endoscopy of the airway in infants and young children. 7 Pediatr 1988;112:1-6.

2 Scott JP, Higenbottam TW, Smyth RL, et al. Transbronchial biopsies in children after heart-lung transplantation.
ben biopsies in children after
Pediatrics 1990;86:698-702.

3 Brain AIJ. The laryngeal mask-a new concept in airway management. Br f Anaesth 1983;55:801-5.

4 Badr A, Tobias JD, Rasmussen GE, Stokes DC, Neblett WW. Bronchoscopic airway evaluation facilitated by the laryngeal mask airway in pediatric patients. Pediatr Pulmonol 1996;21:57-61. 